\title{
Neutralizing potency of COVID-19 vaccines against the SARS-CoV-2 Omicron (B.1.1.529) variant
}

Giuseppe Lippi ( $\nabla$ giuseppe.lippi@univr.it )

Camilla Mattiuzzi

Brandon M. Henry

Short Report

Keywords: SARS-CoV-2, COVID-19, Omicron, Neutralization, Variants

Posted Date: December 28th, 2021

DOI: https://doi.org/10.21203/rs.3.rs-1207848/v1

License: (c) (i) This work is licensed under a Creative Commons Attribution 4.0 International License.

Read Full License

Version of Record: A version of this preprint was published at Journal of Medical Virology on January 5th, 2022. See the published version at https://doi.org/10.1002/jmv.27575. 


\title{
Neutralizing potency of COVID-19 vaccines against the SARS-CoV-2 \\ Omicron (B.1.1.529) variant
}

\author{
Giuseppe Lippi ${ }^{1}$, Camilla Mattiuzzi ${ }^{2}$, Brandon M. Henry ${ }^{3,4}$
}

1. Section of Clinical Biochemistry and School of Medicine, University of Verona, Italy.

2. Service of Clinical Governance, Provincial Agency for Social and Sanitary Services, Trento, Italy.

3. Clinical Laboratory, Division of Nephrology and Hypertension, Cincinnati Children's Hospital Medical Center, Cincinnati, OH, USA

4. Disease Intervention \& Prevention and Population Health Programs, Texas Biomedical Research Institute, San Antonio, Texas, USA

Short title: Post-vaccine neutralization of SARS-CoV-2 Omicron

Word count: $683+1$ Table

Type of article: Short report

Keywords: SARS-CoV-2; COVID-19; Omicron; Neutralization; Variants

\section{Corresponding author:}

Prof. Giuseppe Lippi

Section of Clinical Biochemistry

University Hospital of Verona

Piazzale L.A. Scuro, 10

37134 Verona - Italy

Tel. 0039-045-8122970

Fax. 0039-045-8124308

Email: giuseppe.lippi@univr.it 


\section{Abstract}

Background: We carried out a literature search for summarizing currently published evidence on severe acute respiratory syndrome coronavirus 2 (SARS-CoV-2) Omicron (B.1.1.529) variant neutralizing properties of serum or plasma collected from recipients of coronavirus disease 2019 (COVID-19) vaccines.

Methods: An electronic search was conducted in Medline and Scopus, using the keywords "vaccine" AND "Omicron" OR "B.1.1.529" AND "SARS-CoV-2" AND “neutralization" OR “antibodies", with no language or date limits (i.e., up to December 27, 2021). Studies with complete information on neutralization properties of COVID-19 vaccines against the Omicron variant, with or without the adjunctive effects of booster vaccine doses, were included.

Results: Our final analysis included 10 published studies. In all, decreased neutralisation of SARS-CoV-2 Omicron B.1.1.529 variant was evidenced in postvaccination samples, ranging between -4.3 folds to absence of neutralization compared to an ancestral SARS-CoV-2 strain. In all studies the COVID-19 vaccine booster dose was effective to elicit sustained enhancement of SARS-CoV-2 Omicron B.1.1.529 neutralisation, with such increase being comprised between 10-42 folds compared to the pre-booster period.

Conclusion: Vaccine boosters seem strongly advisable for limiting the risk of SARSCoV-2 Omicron (B.1.1.529) breakthrough infections. 


\section{Introduction}

The recent emergence of a new variant of the severe acute respiratory syndrome coronavirus 2 (SARS-CoV-2) called Omicron (B.1.1.529) has raised paramount concerns in scientific and medical communities due to the presence of several mutations in the spike protein, many of which are located within the receptor binding domain (RBD). ${ }^{1}$ Some of these mutations were found to have a substantial influence on host cells receptor and anti-SARS-CoV-2 antibodies binding, ${ }^{2}$ which may then impact infectivity and neutralizing antibodies escape, thus potentially magnifying the risk of COVID-19 (coronavirus disease 2019) vaccine breakthrough. We have hence carried out a scientific literature search, aimed at summarizing the currently published evidence on Omicron variant neutralizing properties of serum or plasma collected from recipients of COVID-19 vaccines.

\section{Materials and Methods}

We carried out an electronic search in Medline and Scopus, using the keywords "vaccine" AND "Omicron” OR "B.1.1.529" AND "SARS-CoV-2" AND "neutralization" OR “antibodies", with no language or date limits (i.e., up to December 27, 2021). Title, summary and (eventually) full text of all documents identified based on these search criteria were scrutinized, and those reporting complete information on the neutralization properties of COVID-19 vaccines against the SARS-CoV-2 Omicron (B.1.1.529) variant, with or without the adjunctive effects of booster vaccine doses administrated after completing a primary vaccination cycle, were included in our analysis. Due to the wide heterogeneity and scarce comparability of commercial immunoassays for measuring anti-SARS-CoV-2 antibodies, only studies using live or pseudotyped virus neutralization assays were retained. The reference list of all documents was also analyzed for identifying other potentially eligible investigations. 
The following data were retrieved from all included studies: type of vaccine(s) used for primary vaccination, type of assay used for studying serum or plasma neutralizing properties, decreased neutralization of Omicron (B.1.1.529) variant compared to an ancestral strain and/or the currently endemic Delta variant, type of vaccine used for administering the homologous booster COVID-19 vaccine dose and, finally, increased level of Omicron (B.1.1.529) neutralization achieved after administering the booster dose.

\section{Results}

Overall, 37 items could be originally detected based on our search criteria, and 6 more could be identified from the reference lists, thus leading to 43 potentially eligible studies. Nonetheless, 33 of such documents ought to be omitted since they did not fulfil our aforementioned search criteria. Our final analysis could thus only include 10 published studies (Table 1), ${ }^{3-12}$ which used the following vaccines: Pfizer/BioNTech BNT162b2 in 7 studies, Moderna mRNA-1273 in 3 studies, AstraZeneca ChAdOx1, Johnson \& Johnson Ad26.COV2.S and Sinovac CoronaVac in 2 studies, whilst Sinopharm BBIBP-CorV, Novavax NVX-CoV2373 and Sputnik V vaccines were only used in 1 study, respectively. Live and pseudotyped virus neutralization assays were used in 3 and 6 studies, respectively, whilst the remaining study used a hACE2 (angiotensin converting enzyme 2) receptor binding test. Six out of these 10 studies described also the neutralizing activity achieved after administering a homologous vaccine booster dose (Table 1).

In all included studies, a decreased neutralisation of SARS-CoV-2 Omicron B.1.1.529 variant was evidenced in post-vaccination samples, ranging between -4.3 folds to absence of neutralization compared to an ancestral SARS-CoV-2 strain, and between -2.1 folds to absence of neutralization compared to the Delta SARS-CoV-2 
variant, respectively (Table 1). For vaccines used in $\geq 3$ studies, decreased neutralisation of SARS-CoV-2 Omicron B.1.1.529 compared to an ancestral strain was comprised between 22-127 folds in Pfizer/BioNTech BNT162b2 recipients, and between 9-122 in those receiving Moderna mRNA-1273, respectively. In all studies the COVID-19 vaccine booster dose was effective to elicit a sustained enhancement of SARS-CoV-2 Omicron B.1.1.529 neutralisation, with such increase being comprised between 10 and 42 folds compared to the pre-booster period (Table 1).

\section{Conclusions}

The results of this preliminary analysis of currently available research reveal that the neutralizing potency of all COVID-19 vaccines seems to be considerably reduced against the newly emerged SARS-CoV-2 Omicron (B.1.1.529), though the administration of vaccine booster doses is seemingly effective to substantially restore post-immunization efficacy against this new and highly mutated lineage. Vaccine boosters seem hence strongly advisable for limiting the risk of SARS-CoV-2 Omicron (B.1.1.529) breakthrough infections, especially in fragile populations or in those at higher risk of infection (e.g., immunocompromised patients).

\section{FUNDING}

This study received no funding.

\section{CONFLICT OF INTERESTS}

The authors declare that there are no conflicts of interest.

\section{DATA AVAILABILITY STATEMENT}

Data available on request. 


\section{References}

1. Wang L, Cheng G. Sequence analysis of the Emerging Sars-CoV-2 Variant Omicron in South Africa. J Med Virol. 2021 Dec 12. doi: 10.1002/jmv.27516. Epub ahead of print. PMID: 34897752 .

2. Saxena SK, Kumar S, Ansari S, Paweska JT, Maurya VK, Tripathi AK, et al. Characterization of the novel SARS-CoV-2 Omicron (B.1.1.529) Variant of Concern and its global perspective. J Med Virol. 2021 Dec 14. doi: 10.1002/jmv.27524. Epub ahead of print.

3. Dejnirattisai W, Shaw RH, Supasa P, Liu C, Stuart AS, Pollard AJ, et al. Reduced neutralisation of SARS-CoV-2 omicron B.1.1.529 variant by post-immunisation serum. Lancet. 2021 Dec 20:S0140-6736(21)02844-0. doi: 10.1016/S01406736(21)02844-0. Epub ahead of print.

4. Ai J, Zhang H, Zhang Y, Lin K, Zhang Y, Wu J, Wan Y, Huang Y, Song J, Fu Z, Wang H, Guo J, Jiang N, Fan M, Zhou Y, Zhao Y, Zhang Q, Liu Q, Lv J, Li P, Qiu C, Zhang W. Omicron variant showed lower neutralizing sensitivity than other SARS-CoV-2 variants to immune sera elicited by vaccines after boost. Emerg Microbes Infect. 2021 Dec 22:1-24. doi: 10.1080/22221751.2021.2022440. Epub ahead of print. PMID: 34935594.

5. Garcia-Beltran WF, St Denis KJ, Hoelzemer A, Lam EC, Nitido AD, Sheehan ML, et al. mRNA-based COVID-19 vaccine boosters induce neutralizing immunity against SARS-CoV-2 Omicron variant. medRxiv [Preprint]. 2021 Dec 14:2021.12.14.21267755. doi: 10.1101/2021.12.14.21267755.

6. Doria-Rose NA, Shen X, Schmidt SD, O'Dell S, McDanal C, Feng W, et al. Booster of mRNA-1273 Vaccine Reduces SARS-CoV-2 Omicron Escape from Neutralizing 
Antibodies. medRxiv [Preprint]. 2021 Dec 15:2021.12.15.21267805. doi: 10.1101/2021.12.15.21267805.

7. Schmidt F, Muecksch F, Weisblum Y, Silva JD, Bednarski E, Cho A, et al. Plasma neutralization properties of the SARS-CoV-2 Omicron variant. medRxiv [Preprint]. 2021 Dec 13:2021.12.12.21267646. doi: 10.1101/2021.12.12.21267646.

8. Cameroni E, Saliba C, Bowen JE, Rosen LE, Culap K, Pinto D, et al. Broadly neutralizing antibodies overcome SARS-CoV-2 Omicron antigenic shift. bioRxiv [Preprint]. 2021 Dec 14:2021.12.12.472269. doi: 10.1101/2021.12.12.472269.

9. Lu L, Mok BW, Chen LL, Chan JM, Tsang OT, Lam BH, et al. Neutralization of SARS-CoV-2 Omicron variant by sera from BNT162b2 or Coronavac vaccine recipients. Clin Infect Dis. 2021 Dec 16:ciab1041. doi: 10.1093/cid/ciab1041. Epub ahead of print.

10. Cele S, Jackson L, Khan K, Khoury DS, Moyo-Gwete T, Tegally H, et al. SARSCoV-2 Omicron has extensive but incomplete escape of Pfizer BNT162b2 elicited neutralization and requires ACE2 for infection. medRxiv [Preprint]. 2021 Dec 11:2021.12.08.21267417. doi: 10.1101/2021.12.08.21267417.

11. Muik A, Lui BG, Wallisch AK, Bacher M, Muehl J, Reinholz J, et al. Neutralization of SARS-CoV-2 Omicron pseudovirus by BNT162b2 vaccine-elicited human sera. medRxiv [Preprint] $2021 \quad$ Dec 23:2021.12.22.21268103; doi: 10.1101/2021.12.22.21268103.

12. Mallory R, Formica N, Pfeiffer S, Wilkinson B, Marcheschi A, Albert G, et al. Immunogenicity and Safety Following a Homologous Booster Dose of a SARSCoV-2 recombinant spike protein vaccine (NVX-CoV2373): A Phase 2 Randomized Placebo-Controlled Trial. medRxiv [Preprint] 2021 Dec 25:2021.12.23.21267374; doi: 0.1101/2021.12.23.21267374. 
Table 1. Neutralizing potency of coronavirus disease 2019 (COVID-19) vaccines against the severe acute respiratory syndrome coronavirus 2 (SARS-CoV-2) Omicron (B.1.1.529) variant

\begin{tabular}{|c|c|c|c|c|c|}
\hline \multirow[b]{2}{*}{ Authors } & \multicolumn{3}{|c|}{ Primary vaccination } & \multicolumn{2}{|c|}{ Booster vaccine dose } \\
\hline & Vaccines; sample collection & $\begin{array}{l}\text { Neutralization } \\
\text { assay }\end{array}$ & Reduced Omicron neutralization (folds) & $\begin{array}{l}\text { Vaccines; sample } \\
\text { collection }\end{array}$ & $\begin{array}{c}\text { Increased Omicron } \\
\text { neutralization after } \\
\text { booster (folds) }\end{array}$ \\
\hline $\begin{array}{l}\text { Dejnirattisai W } \\
\text { et al., } 2021^{3}\end{array}$ & $\begin{array}{c}2 \times \text { ChAdOx } 1 \text { or } 2 \times \text { BNT162b } 2 ; \\
\sim 28 \text { days }\end{array}$ & $\begin{array}{l}\text { Live virus } \\
\text { neutralization assay }\end{array}$ & $\begin{array}{l}\text { Vs. ancestral strain: ChAdOx1: } \downarrow \times 13.3 \text {; } \\
\text { BNT162b2: } \downarrow \times 29.8 \\
\text { Vs. delta variant: ChAdOx1: } \downarrow \times 5.2 \text {; } \\
\text { BNT162b2: } \downarrow \times 25.1\end{array}$ & No & - \\
\hline $\begin{array}{l}\text { Ai J et al., } \\
2021^{4}\end{array}$ & $2 \times$ BBIBP-CorV; $\sim 14$ days & $\begin{array}{l}\text { Pseudotyped virus } \\
\text { neutralization assay }\end{array}$ & $\begin{array}{l}\text { Vs. ancestral strain: BBIBP-CorV: } \downarrow \times 11.6 \text {; } \\
\text { Vs. delta variant: BBIBP-CorV: } \downarrow \times 7.9 \\
\end{array}$ & $\begin{array}{l}1 \times \text { BBIBP-CorV; } 14- \\
28 \text { days }\end{array}$ & BBIBP-CorV: $\uparrow \times 9.6$ \\
\hline $\begin{array}{l}\text { Garcia-Beltran } \\
\text { WF et al., } 2021^{5}\end{array}$ & $\begin{array}{l}2 \times \mathrm{BNT} 162 \mathrm{~b} 2 \text { or } 2 \times \text { mRNA-1273; } \\
<3 \text { months }\end{array}$ & $\begin{array}{l}\text { Pseudotyped virus } \\
\text { neutralization assay }\end{array}$ & $\begin{array}{l}\text { Vs. ancestral strain: BNT162b2: } \downarrow \times 43 \text {; } \\
\text { mRNA-1273: } \downarrow \times 122\end{array}$ & $\begin{array}{l}\text { 1× BNT162b2 or } 1 \times \\
\text { mRNA-1273; }<3 \\
\text { months }\end{array}$ & $\begin{array}{l}\text { BNT162b2 } \uparrow \times 27 \\
\text { mRNA-1273: } \uparrow \times 19\end{array}$ \\
\hline $\begin{array}{l}\text { Doria-Rose NA. } \\
\text { et al., } 2021^{6}\end{array}$ & 2×mRNA-1273; 14 days & $\begin{array}{l}\text { Pseudotyped virus } \\
\text { neutralization assay }\end{array}$ & Vs. ancestral strain: mRNA-1273: $\downarrow \times 8.9$ & $\begin{array}{l}1 \times \text { mRNA-1273; } 14 \\
\text { days }\end{array}$ & mRNA-1273: $\uparrow \times 12.6$ \\
\hline $\begin{array}{l}\text { Schmidt F et } \\
\text { al., } 2021^{7}\end{array}$ & $\begin{array}{c}2 \times \text { BNT162b } 2 \text { or } 1 \times \\
\text { Ad26.COV2.S; } \sim 1.3 \text { month }\end{array}$ & $\begin{array}{l}\text { Pseudotyped virus } \\
\text { neutralization assay }\end{array}$ & $\begin{array}{l}\text { Vs. ancestral strain: BNT162b2: } \downarrow \times 127 \text {; } \\
\text { Ad26.COV2.S: No neutralization }\end{array}$ & $\begin{array}{l}\times \text { BNT162b2 or } 1 \times \\
\text { Ad26.COV2.S; } 28 \\
\text { days }\end{array}$ & $\begin{array}{l}\text { BNT162b2: } \uparrow \times 42.1 \\
\text { Ad26.COV2.S: } \\
\text { unavailable }\end{array}$ \\
\hline $\begin{array}{l}\text { Cameroni e et } \\
\text { al., } 2021^{8}\end{array}$ & $\begin{array}{l}2 \times \text { BNT162b2 or } 2 \times \text { mRNA- } 1273 \\
\text { or } 2 \times \text { ChAdOx } 1 \text { or } 1 \times \\
\text { Ad26.COV2.S; } 2 \times \text { Sinovac; } 2 \times \\
\text { Sptunik; } 14-28 \text { days } \\
\end{array}$ & $\begin{array}{l}\text { Pseudotyped virus } \\
\text { neutralization assay }\end{array}$ & $\begin{array}{l}\text { Vs. ancestral strain: BNT162b2: } \downarrow \times 44 \text {; } \\
\text { mRNA-1273: } \downarrow \times 33 \text {; ChAdOx1: } \downarrow \times 36 \text {; } \\
\text { Ad26.COV2.S: No neutralization; Sinovac: No } \\
\text { neutralization; Sptunik: No neutralization }\end{array}$ & No & 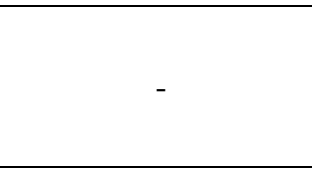 \\
\hline $\begin{array}{l}\text { Lu L et al., } \\
2021^{9}\end{array}$ & $\begin{array}{l}2 \times \text { BNT162b2 or } 2 \times \text { Sinovac; } \sim 1 \\
\text { month }\end{array}$ & $\begin{array}{l}\text { Live virus } \\
\text { neutralization assay }\end{array}$ & $\begin{array}{l}\text { Vs. ancestral strain: BNT162b2: } \downarrow \times 38.7 \text {; } \\
\text { Sinovac } \downarrow \times 4.3 \\
\text { Vs. delta variant: BNT162b2: } \downarrow \times 21.0 \text {; } \\
\text { Sinovac } \downarrow \times 2.1\end{array}$ & No & - \\
\hline $\begin{array}{l}\text { Cele } \mathrm{S} \text { et al., } \\
2021^{10}\end{array}$ & $2 \times$ BNT162b2; 10-63 days & $\begin{array}{c}\text { Live virus } \\
\text { neutralization assay }\end{array}$ & Vs. ancestral strain: BNT162b2: $\downarrow \times 22$ & No & - \\
\hline $\begin{array}{l}\text { Muik A et al., } \\
2021^{11}\end{array}$ & $2 \times \mathrm{BNT} 162 \mathrm{~b} 2 ; \sim 21$ days & $\begin{array}{l}\text { Pseudotyped virus } \\
\text { neutralization assay }\end{array}$ & $\begin{array}{l}\text { Vs. ancestral strain: BNT162b2: } \downarrow \times 22.8 \\
\text { Vs. delta variant: BNT162b2: } \downarrow \times 10.4\end{array}$ & $\begin{array}{c}1 \times \mathrm{BNT} 162 \mathrm{~b} 2 ; \sim 1 \\
\text { month }\end{array}$ & BNT162b2: $\uparrow \times 23.4$ \\
\hline $\begin{array}{l}\text { Mallory R et al, } \\
2021^{12}\end{array}$ & $2 \times N V X-C o V 2373 ; ~ 14$ days & $\begin{array}{l}\text { hACE2 receptor } \\
\text { binding test }\end{array}$ & $\begin{array}{l}\text { Vs. ancestral strain: NVX-CoV2373: } \downarrow \times 8.2 \\
\text { Vs. delta variant: NVX-CoV2373: } \downarrow \times 2.8\end{array}$ & $\begin{array}{c}1 \times \mathrm{NVX}-\mathrm{CoV} 2373 \\
\sim 28 \text { days }\end{array}$ & $\begin{array}{l}\text { NVX-CoV2373: } \\
\uparrow \times 14.8\end{array}$ \\
\hline
\end{tabular}

Vaccines legend: AstraZeneca ChAdOx1 (adenovirus-based); Johnson \& Johnson Ad26.COV2.S (adenovirus-based); Moderna mRNA-1273 (mRNA-based); Novavax NVXCoV2373 (recombinant spike protein vaccine); Pfizer/BioNTech BNT162b2 (mRNA-based); Sinopharm BBIBP-CorV (inactivated); Sinovac CoronaVac (inactivated); Sputnik

$\mathrm{V}$ vaccine (adenovirus-based). 